\title{
DRENAGEM ENDOSCÓPICA TRANSMURAL DE PSEUDOCISTO PANCREÁTICO: resultados a longo prazo
}

\author{
Rone Antônio Alves de ABREU1', Joaquim Alves CARVALHO Jr.², Filinto Anibal Alagia \\ VAZ1 \\ Luiz Hirotoshi OTA ${ }^{3}$ e Manlio Basílio SPERANZINI ${ }^{4}$
}

RESUMO - Racional - Os pseudocistos pancreáticos são complicações relativamente comuns em pacientes adultos com pancreatite. Objetivo - Avaliar os resultados a longo prazo da drenagem endoscópica transmural, estabelecendo seu papel no manejo do pseudocisto pancreático. Métodos - Foram estudados 14 pacientes com pseudocisto de pâncreas, cuja principal queixa à apresentação foi dor no andar superior do abdome e massa abdominal palpável, submetidos a cistogastrostomia $(\mathrm{n}=12)$ e cistoduodenostomia $(\mathrm{n}=2)$, acompanhados clinicamente e com tomografia computadorizada de abdome por até 51 meses. A colangiopancreatografia endoscópica retrógrada era tentada em todos os casos para estudo do ducto pancreático e classificação dos cistos. Resultados - A pancreatite crônica alcoólica agudizada foi responsável por 10 casos $(71,5 \%)$ e a biliar por 4 (28,5\%). As duas formas de drenagens (cistogastrostomia e cistoduodenostomia) endoscópicas foram efetivas. Não houve mudança na conduta terapêutica proposta; em dois pacientes a migração da órtese para o interior do pseudocisto, no momento da inserção, foi a principal complicação, sendo possível sua retirada no mesmo ato, com o uso da cesta de Dormia, sob o auxílio de fluoroscopia. Não houve mortalidade, nem recidiva até o momento. O tempo médio de permanência hospitalar foi de 3 dias. Conclusão - A drenagem endoscópica transmural se apresentou como terapêutica eficaz, com baixo índice de complicações, mortalidade nula e pequeno tempo de internação hospitalar

DESCRITORES - Pseudocisto pancreático. Drenagem. Contenedores. Endoscopia gastrointestinal.

\section{INTRODUÇÃO}

Complicação relativamente comum em pacientes adultos, com diagnóstico de pancreatite, os pseudocistos pancreáticos ocorrem em 16\%-50\% dos casos de pancreatite aguda e em $20 \%-40 \%$ das pancreatites crônicas ${ }^{(2,14)}$. As lesões císticas pancreáticas foram classificados de acordo com D'Egidio e Schein, em 1992, em tipo I: pósnecróticos, que surgem após episódios de pancreatite aguda, sem anormalidades identificáveis nos ductos pancreáticos e, raramente, comunicando-se com um canal do pâncreas; tipo II: ocorrem em casos de agudização de pancreatite crônica, reconhecida pelas alterações dos ductos pancreáticos, com os quais freqüentemente os pseudocistos se comunicam; tipo III: pseudocistos de retenção da pancreatite crônica calcificante, comprovada pelas alterações ductais características, ou presença de cálculos intracanaliculares (calcificações) ${ }^{(3,9,10)}$. O tratamento cirúrgico já está estabelecido, apesar do crescente aumento de técnicas menos invasivas como as drenagens endoscópica, percutânea e guiada por ultra-sonografia $^{(12)}$.

O presente estudo visou avaliar os resultados a longo prazo da drenagem endoscópica transmural.

\section{MÉTODOS}

Foram estudados no Conjunto Hospitalar do Mandaqui, São Paulo, SP, de novembro de 2000 a fevereiro de 2005, 14 pacientes com pseudocisto de pâncreas (Figura 1), que preencheram os seguintes critérios de inclusão: 6 semanas de tratamento clínico sem regressão; tamanho maior ou igual a $4 \mathrm{~cm}$; distância entre parede gastroduodenal e parede do pseudocisto menor ou igual a $1 \mathrm{~cm}$; ausência de processo neoplásico associado; aparecimento de complicações e/ou persistência dos sintomas e abaulamento evidente da parede gastroduodenal.

Quanto ao gênero, 71,5\% (10/14) dos pacientes eram do sexo masculino e $28,5 \%$ (4/14) do feminino. A idade variou de 28 a 57 anos, com média de 45 anos. Pancreatite

\footnotetext{
Setor de Endoscopia Digestiva do Serviço de Cirurgia Geral e do Aparelho Digestivo do Conjunto Hospitalar do Mandaqui, São Paulo, SP.

Conjunto Hospitalar do Mandaqui; ${ }^{2}$ Santa Casa de Misericórdia de São Paulo; ${ }^{3}$ Disciplina de Cirurgia Torácica da Universidade Federal de São Paulo, São Paulo, SP; ${ }^{4}$ Faculdade de Medicina do ABC, Santo André, SP.

Correspondência: Dr. Rone Antônio Alves de Abreu - Rua Voluntários da Pátria, 3880 - apt.144-A - Santana. São Paulo, SP. E-mail: roneabreu@uol.com.br
} 


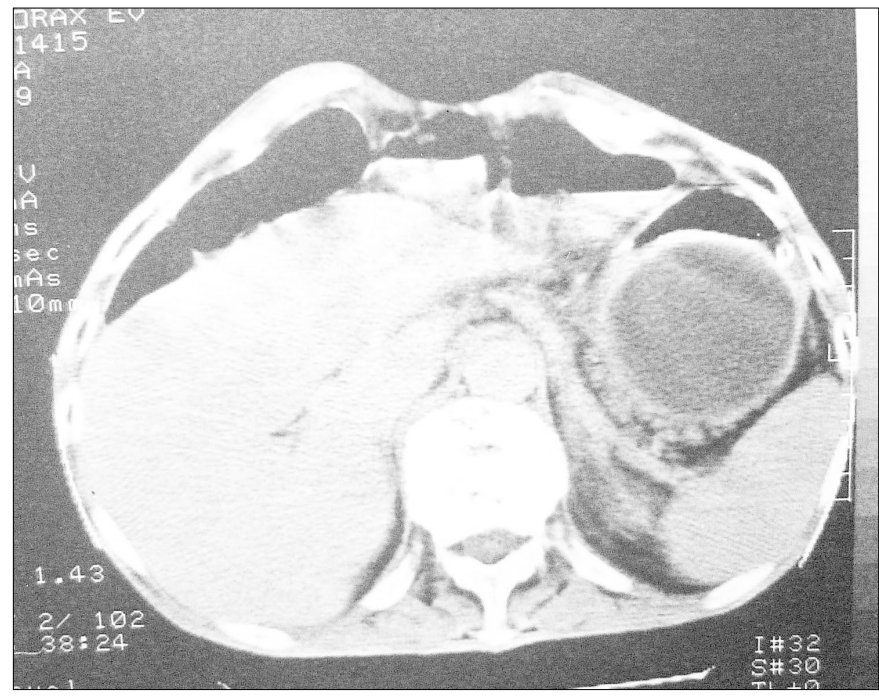

FIGURA 1. TC de abdome: pseudocisto de pâncreas

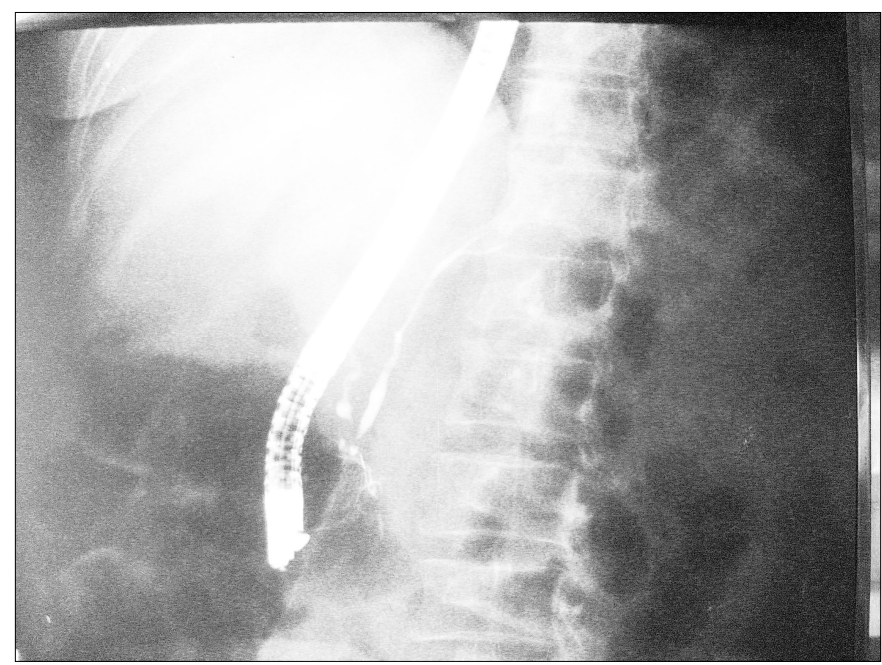

FIGURA 2. Pancreatografia: ausência de comunicação do Wirsung com o pseudocisto

TABELA 1. Característica da amostra

\begin{tabular}{lcc}
\hline & $\mathbf{n}$ & $\%$ \\
\hline Sexo & & \\
masculino & 10 & 71,5 \\
Feminino & 4 & 28,5 \\
Causa da pancreatite & & \\
$\quad$ alcoólica crônica agudizada & 10 & 71,5 \\
$\quad$ biliar & 4 & 28,5 \\
Momento da drenagem na evolução & & \\
6 semanas & 12 & 85,7 \\
após 6 semanas & 2 & 14,2 \\
Classificação dos cistos & & \\
$\quad$ tipo I & 9 & 64,2 \\
tipo II & 1 & 7,1 \\
não classificado & 4 & 28,5 \\
Total & 4 & 100 \\
\hline
\end{tabular}

crônica alcoólica foi responsável pela etiologia em 71,5\% (10/14) dos doentes e a biliar em 28,5\% (4/14). Dois pacientes ingressaram no estudo com diagnóstico já firmado, com 18 e 36 meses de evolução após o episódio de internação por pancreatite aguda. Todos foram examinados por tomografia computadorizada de abdome, com cortes para estudo pancreático, visando excluir hipertensão portal segmentar e pseudoaneurisma de artéria visceral, caracterizar o conteúdo fluido do pseudocisto, bem como afastar necrose no seu interior (Figura 1). A pancreatografia endoscópica retrógrada para estudo do ducto (Figura 2), foi tentada em todos os casos, não sendo possível em quatro pacientes (Tabela 1).

\section{Técnica}

Os seguintes passos foram padronizados:

1. Antibioticoprofilaxia (ceftriaxona $-1 \mathrm{~g}$ intravenosa a cada 12 horas, mantida por 48 horas após o procedimento);

2. Sedação foi obtida com midazolan na dosagem de $0,03 \mathrm{mg} / \mathrm{kg} /$ peso, equivalente a $2,0 \mathrm{mg}$ para cada $70 \mathrm{~kg}$, associados a meperidina $20 \mathrm{mg}$, adicionando-se doses complementares no decurso do procedimento de acordo com a necessidade;

3. Escopolamina, $40 \mathrm{mg}$ intravenosa, para obter atonia duodenal;

4. A endoscopia foi realizada com o aparelho Olympus ${ }^{\bigotimes}$ modelo TJF10, canal de trabalho de 4,2 mm, precedida da pancreatografia retrógrada endoscópica, para estudo do ducto e classificação do cisto;

5. Identificada a área mais abaulada do segmento gastroduodenal, observada à tomografia e à endoscopia;

6. Punção da área eleita com agulha de esclerose endoscópica (10 x $4 \mathrm{~mm}$ ), para confirmação da proximidade com o cisto;

7. Fistulização do trato gastrointestinal (duodeno ou estômago) com o pseudocisto por meio de alça de polipectomia semi-aberta conectada a eletro-cautério - WEN ${ }^{\bigotimes}$, modelo SS501, utilizando-se apenas a função de coagulação, monopolar, na intensidade 30 watts;

8. Canulação imediata do cisto com cateter de colangiografia e injeção de contraste iodado não-iônico (300 mg iodo/mL; $612 \mathrm{mg}$ iopamidol/mL) (Figura 3), para documentação e certificação do

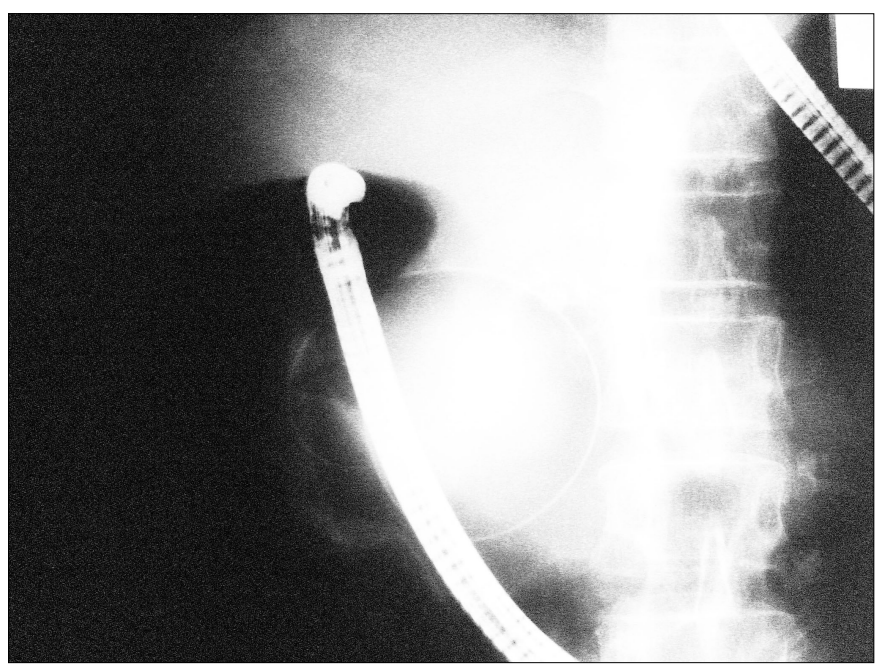

FIGURA 3. CPRE: fio guia no interior do pseudocisto pancreático 
cisto, com colocação de órtese (stent) biliar de calibre $10 \mathrm{Fr}$, curta ( $6 \mathrm{~cm}$, no máximo), com duas farpas, uma em cada extremidade (Figura 4 e 5). Observando-se a drenagem do fluido pelo stent, dava-se por encerrado o procedimento.

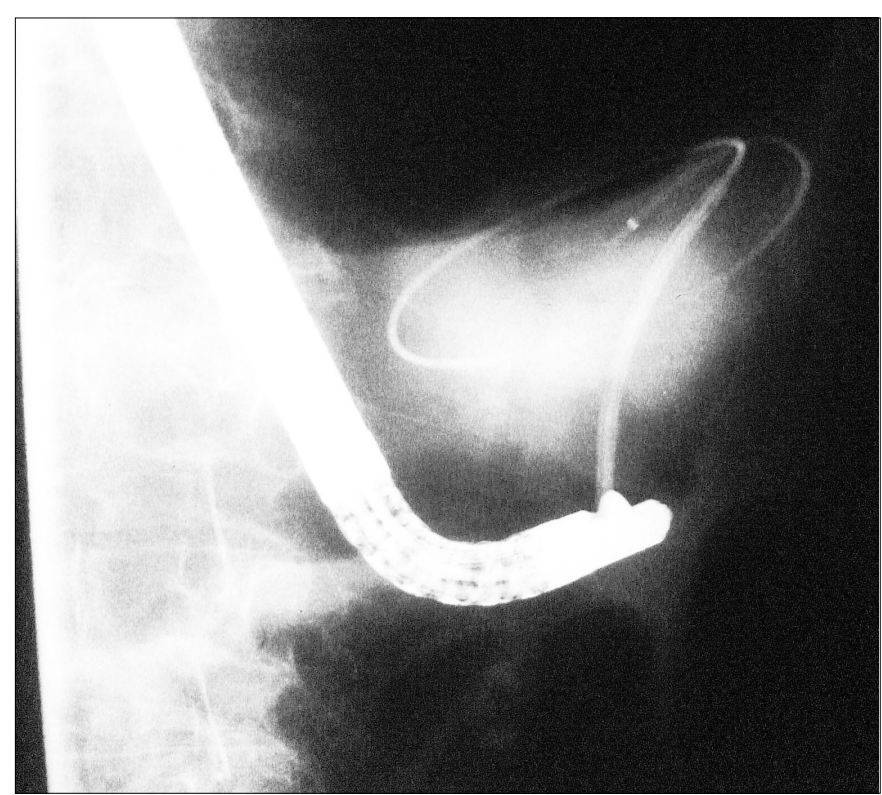

FIGURA 4. CPRE: locação da endoprótese através da parede do estômago

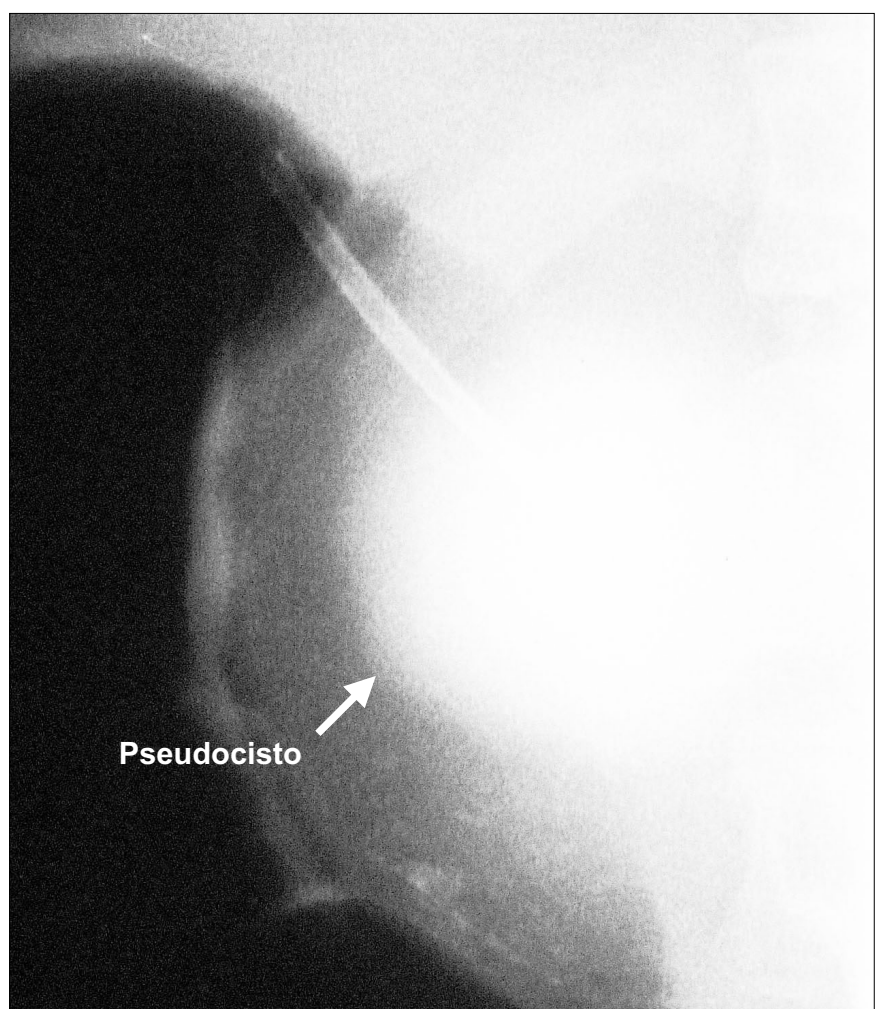

FIGURA 5. Aspecto final: órtese comunicando o pseudocisto com TGI
A órtese foi retirada endoscopicamente, 4 a 6 semanas após avaliação clínica e novo estudo tomográfico (Figura 6).

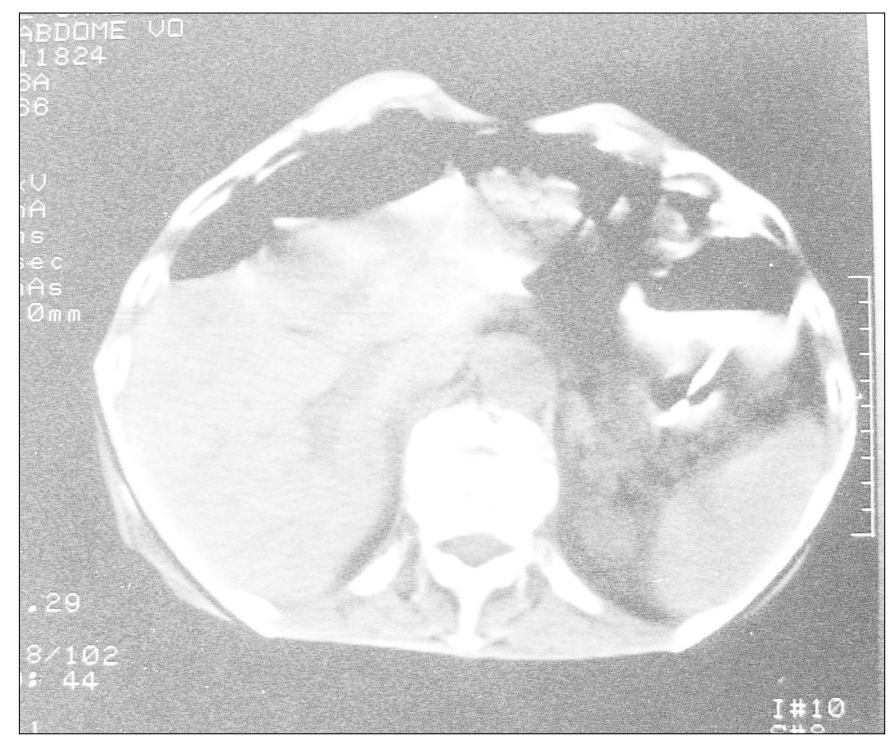

FIGURA 6. TC de abdome no $14^{\circ}$ dia pós-drenagem (controle do paciente da Figura 1): órtese (seta) comunicando o pseudocisto com estômago

\section{Seguimento}

A dor abdominal e os sintomas obstrutivos foram avaliados clinicamente e a regressão do pseudocisto foi confirmada por tomografia computadorizada abdominal 4 a 6 semanas após.

\section{RESULTADOS}

No quadro clínico, a dor abdominal no andar superior do abdome foi o sintoma predominante em $92,8 \%$ (13/14). A icterícia se apresentou às custas da fração direta, sugerindo compressão extrínseca, enquanto a anemia se mostrou do tipo megaloblástica. Dois casos apresentaram ascite pancreática (14,3\%) (Tabela 2).

\section{TABELA 2. Quadro clínico}

\begin{tabular}{lcc}
\hline Sinais e sintomas & $\mathbf{n}$ & $\%$ \\
\hline Dor abdominal & 13 & 92,8 \\
Massa abdominal & 10 & 71,4 \\
Perda de peso & 5 & 35,7 \\
Náusea e vômitos & 5 & 35,7 \\
Esteatorréia & 3 & 21,4 \\
Icterícia & 3 & 21,4 \\
Anemia* & 3 & 21,4 \\
Ascite & 2 & 14,2 \\
Sinais de peritonite & 1 & 7,1 \\
\hline
\end{tabular}

*hematócrito menor ou igual a $30 \%$

Ao exame de tomografia computadorizada de abdome foram diagnosticados 16 pseudocistos, sendo: 12 pacientes com 1 pseudocisto 
e 2 pacientes com 2. O tamanho do pseudocisto variou de 8 a 22 $\mathrm{cm}$ (média de $16 \mathrm{~cm}$ ), assim distribuídos: 10 estavam localizados na cabeça, 4 no corpo e 2 na cauda do pâncreas. Em todos os casos identificou-se abaulamento na parede gástrica ou duodenal.

Todos foram tratados por endoscopia, sendo realizados 15 procedimentos endoscópicos. A pancreatografia foi obtida em $71,4 \%$ (10/14) dos pacientes, sendo $9(64,2 \%)$ do tipo I e $1(7,1 \%)$ do tipo II. Em 12 pacientes o abaulamento gástrico foi mais evidente, sendo realizada drenagem cistogástrica; 2 casos apresentavam abaulamento com predomínio na região duodenal, favorecendo à drenagem cistoduodenal. Não houve necessidade de mudança na técnica.

Nos 15 procedimentos endoscópicos realizados ocorreram 3 complicações em dois pacientes. Nestes, no momento da colocação da órtese, houve migração para o interior do pseudocisto, a qual foi retirada com a cesta de Dormia sob controle fluoroscópico. Em um caso de drenagem para o duodeno, durante a manobra de retirada da órtese, provocou-se inadvertidamente ampliação do orifício fistuloso, impossibilitando a colocação e manutenção de nova órtese. Tendo ocorrido esvaziamento quase total do conteúdo do cisto, optou-se por mantê-lo em observação. No $4^{\circ}$ dia de seguimento a paciente apresentou febre e novamente aumento do volume abdominal. Nova CT de abdome demonstrou que o pseudocisto havia recidivado. Em nova intervenção endoscópica notou-se oclusão de mais de $90 \%$ do orifício fistuloso para o duodeno e, evidente abaulamento gástrico. Realizou-se nova cistogastrostomia e locação de stent $10 \mathrm{Fr}$, com saída de líquido de odor fétido, que foi aspirado. A paciente evoluiu satisfatoriamente, recebendo alta hospitalar em 4 dias.

O tempo médio de permanência hospitalar foi de 3 dias.

Não houve obstrução dos stents, que foram retirados entre a $4^{\mathrm{a}}$ e a $6^{\mathrm{a}}$ semana, com média de 38 dias. Em um paciente no controle radiológico da $6^{\mathrm{a}}$ semana, comprovou-se a saída espontânea da órtese, que se encontrava na ampola retal, sendo retirada manualmente (Figura 7). Não houve nenhuma complicação hemorrágica ou perfuração para peritônio livre.

A maioria dos doentes com pancreatite alcoólica teve remissão da dor abdominal (10/12), porém dois pacientes mantiveram-se com opióides para controle da dor. Os pacientes cujos pseudocistos eram de etiologia biliar, foram submetidos posteriormente a colecistectomia e permanecem assintomáticos.

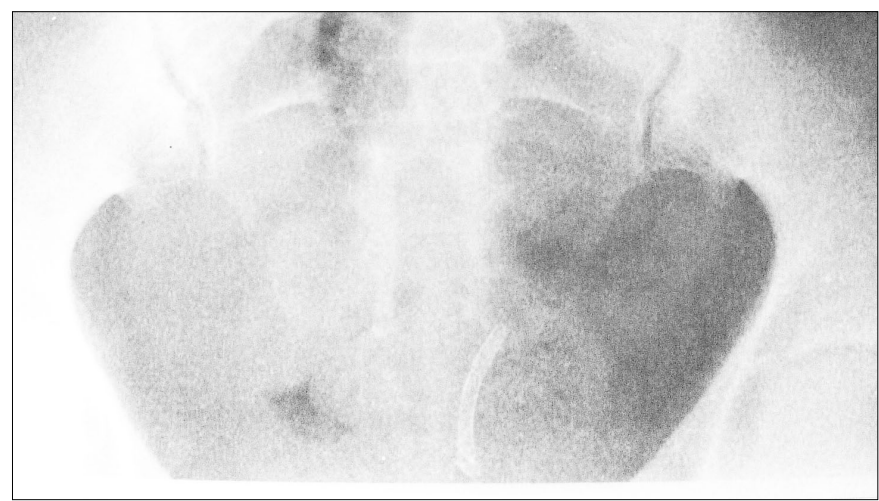

FIGURA 7. Migração distal: órtese no reto
Os dois casos de ascite pancreática tiveram regressão da mesma após a drenagem endoscópica.

No seguimento em ambulatório os pacientes evoluíram sem recidivas, em período que variou de 12 a 51 meses, com média de 32 meses. Não houve mortalidade relacionada com o procedimento. No seguimento dois pacientes vieram a falecer: um por infarto agudo do miocárdio e outro por abdome agudo obstrutivo, 6 e 18 meses, respectivamente, após as drenagens.

\section{DISCUSSÃO}

Nesta casuística, em 10/14 pacientes a pancreatite crônica foi responsável pelos pseudocistos e massa abdominal palpável e dor foram os sintomas principais. A etiologia mais freqüente dos pseudocistos é atribuída à pancreatite crônica alcoólica ${ }^{(2,4,11,14,17,20)}$ e entre os sinais e sintomas observados predominam a massa abdominal palpável e a dor em andar superior do abdome ${ }^{(3,10)}$. A etiologia predominante, no entanto, pode variar em função de peculiaridades da casuística. Assim, para SHARMA et al. ${ }^{(19)} \mathrm{em}$ sua série de 38 casos, a principal etiologia foi a pancreatite biliar em $50 \%$ dos casos, diferindo sobremaneira da presente série.

A principal indicação de drenagem foi a persistência de dor abdominal (em 98,5\% dos casos), o que está de acordo com O'MALLEY et al. ${ }^{(17)}$ que relataram que este sintoma foi o responsável em 93,4\%.

$\mathrm{Na}$ literatura ainda há controvérsias quanto a melhor forma de abordagem terapêutica para os pseudocistos pancreáticos.

O uso de antibióticos profiláticos não tem sua indicação precisa estabelecida, havendo quem o preconize ${ }^{(6,15)}$, enquanto outros preferem seu uso terapêutico somente após a ocorrência de sinais de infecção do pseudocisto ${ }^{(19)}$. Neste estudo utilizou-se antibioticoprofilaxia sistematicamente, percebendo-se quadro infeccioso em apenas um caso, justamente na paciente em que foi realizada apenas a cistoduodenostomia sem manter a órtese. Acredita-se que a não-fixação da órtese tenha favorecido o fechamento precoce do orifício fistuloso, dificultando a drenagem do pseudocisto que se refez, infectando-se em seguida. Após a reabordagem endoscópica e nova drenagem, com uma órtese de $10 \mathrm{Fr}$, agora para o estômago, a paciente teve uma boa evolução, com resolução da infecção e do pseudocisto.

A ecoendoscopia tem sido usada para decidir o local ideal para punção, pois pode avaliar a presença de vasos, identifica pseudoaneurismas e pode distinguir pseudocistos de cistos neoplásicos ${ }^{(13,20)}$. Entretanto, para alguns autores ${ }^{(15)}$, a ecoendoscopia não é necessária. SHARMA et al. ${ }^{(19)}$ afirmaram que se houver seleção criteriosa dos pacientes com história prévia de pancreatite aguda, crônica ou pancreatite traumática e que o pseudocisto imprima evidente abaulamento do estômago ou duodeno, não há necessidade de se fazer a ecoendoscopia. Opinião esta compartilhada pelos autores deste estudo respaldados pela evolução satisfatória dos pacientes da casuísticas.

No presente estudo havia, à endoscopia, evidente abaulamento na parede posterior do estômago ou da primeira porção duodenal de todos os pacientes. Segundo alguns autores a drenagem transmural, principalmente a cistogastrostomia, apresenta elevados riscos de complicações, especialmente sangramento ${ }^{(13,20)}$. 
BECKINGHAM et al. ${ }^{(3)}$ referiram hemorragia em $11,7 \%$ (4/34) dos pacientes, sendo que 1 foi submetido a cirurgia de urgência para coibir o sangramento. Esses autores realizavam cistoenterostomias amplas, através da utilização de papilótomos de $10 \mathrm{~mm}$ para permitir ampla drenagem, aumentando assim, em nosso entendimento a probabilidade de hemorragias. CREMER et al. ${ }^{(8)}$ teve complicação hemorrágica em $9 \%$ dos pacientes (1/11). Nesta série, após a realização da fístula, retirou-se a alça de polipectomia e procedeu-se à canulação imediata com o cateter de colangiografia, orientados pela saída inicial de fluido pelo orifício fistuloso. Há alças de polipectomias desmontáveis que permitem a passagem do fio-guia após a introdução do cateter teflonado no interior do cisto, tornando mais fácil essa etapa do procedimento. Apesar de não se ter utilizado deste recurso, conseguiu-se a recanulação em todos os casos. Não houve nenhuma complicação hemorrágica, atribuída ao fato de a abertura na parede ser apenas o suficiente para locação da órtese de 10 Fr. Têm-se a impressão de que, diferentemente dos abscessos nos quais deve-se fazer ampla drenagem, nos pseudocistos isso não seria necessário. É possível que a órtese pelas suas dimensões, provoque fluxo unidirecional, do pseudocisto para a luz do estômago ou duodeno. Caso o orifício fistuloso fosse maior facilitaria a contaminação do pseudocisto, em função da maior entrada de conteúdo gastroduodenal para dentro da cavidade. Outro fato a salientar é o comprimento da órtese, sempre o mais curto possível, uma vez que a resistência ao fluxo do conteúdo do cisto é diretamente proporcional ao comprimento da órtese e inversamente proporcional ao quadrado do seu raio [Resistência $=(\mathrm{k}) \mathrm{x}$ (comprimento da órtese) x $1 /$ diâmetro $\left.^{2}\right]$. Com esta órtese foi possível promover o esvaziamento dos pseudocistos e, mantendo-se a drenagem por 1 mês, permitiu a coalescência de suas paredes e sua oclusão total por fibrose.

Houve apenas duas complicações no momento da drenagem, ambas por migração da órtese para o interior do cisto e que foram recuperadas com a cesta de Dormia sob o controle da fluoroscopia. É possível que tal complicação pudesse ser minimizada com a utilização de próteses em duplo "pigtail". No entanto, a retirada da órtese no interior do cisto, reforça a idéia recente na literatura da possibilidade de realização de necrosectomia através da manipulação endoscópica. Estudo recente $^{(5)}$ vem preconizando, quando possível, a dilatação da "fístula" com cateter balonado, a fim de permitir a passagem de duas próteses, visando melhorar a drenagem. Apesar de tal recomendação, CAHEN et al. ${ }^{(5)}$ utilizaram, inicialmente, prótese única em $76 \%$ dos seus pacientes, com bons resultados.

Em um paciente adiou-se por 4 semanas o procedimento terapêutico, face à delgada parede do pseudocisto no momento em que foi encaminhado para o tratamento. Considerou-se importante aguardar a adequada maturação do cisto, obtendose paredes mais espessas e aderência ao estômago ou duodeno mais firmes e confiáveis. Com isso, evitaram-se possíveis complicações, tais como extravasamento para peritônio livre e posterior peritonite.

Os resultados deste estudo são considerados bons quando comparados com os da literatura. Della LIBERA et al. ${ }^{(11)} \mathrm{em}$ série de 30 pacientes, apresentaram $70 \%$ de eficácia no total dos procedimentos, cujo índice de sucesso se assemelha ao obtido, se considerar somente suas drenagens combinadas (transmural e transpapilar associadas). O número de complicações foi semelhante, porém de maior gravidade, como pancreatite, sangramento, perfuração duodenal e pneumoperitônio. BECKINGHAM et al. ${ }^{(3)}$ relataram $71 \%$ de êxito com $14,5 \%$ de morbidade, incluindo sangramentos e perfuração com necessidade de intervenção cirúrgica. $\mathrm{O}$ tratamento cirúrgico convencional também não está isento de possíveis complicações. Assim, O'MALLEY et al. ${ }^{(17)}$ obtiveram com este procedimento, taxa de morbidade de $37 \%$ e mortalidade de $4 \%$.

Nos dois pacientes em que havia dois pseudocistos à tomografia, estes intercomunicavam-se. Realizou-se drenagem apenas do cisto maior e os menores desapareceram espontaneamente após este procedimento, o mesmo ocorrendo com os dois pacientes com ascite pancreática que tiveram resolução espontânea da ascite após o tratamento endoscópico dos cistos.

Os trabalhos descritos na literatura para tratamento de pseudocisto - endoscópico, por punção ou cirurgia - não mencionam a taxa de permanência hospitalar após os procedimentos. Na presente casuística a média de permanência hospitalar pós-operatória foi de 3 dias. O curtíssimo tempo de internação trouxe inúmeros benefícios para a instituição e para os pacientes, tais como: redução dos gastos hospitalares, menor taxa de ocupação e menor tempo de convalescença dos pacientes após as intervenções.

\section{CONCLUSÃO}

A drenagem endoscópica transmural do pseudocisto pancreático, nos casos com abaulamento evidente do estômago ou duodeno, pode ser empregada como primeira escolha, desde que respeitados rigorosamente os critérios de inclusão e quando não houver dúvida diagnóstica quanto à natureza do pseudocisto. Acredita-se que seja importante escolher o local mais adequado onde se procederá a cistostomia e em que momento, sendo necessário aguardar a maturação do cisto a fim de evitar perfuração em peritônio livre.

Com estes cuidados, esta técnica se apresentou como terapêutica eficaz, apresentando baixo índice de complicações, mortalidade nula, possibilitando curta permanência hospitalar. 
Abreu RAA, Carvalho Jr JA, Vaz FAA, Ota LH, Speranzini MB. Transmural endoscopy drainage of pancreatic pseudocyst: long-term outcome. Arq Gastroenterol. 2007;44(1):29-34.

ABSTRACT - Background - Pancreatic pseudocysts are relatively common complications of pancreatitis in adults. Objective - To evaluate the long-term results from transmural endoscopic drainage and thus to establish its role in managing pancreatic pseudocyst. Methods - Fourteen patients with pancreatic pseudocyst were studied. Their main complaint was pain in the upper levels of the abdomen. They presented palpable abdominal mass and underwent cystogastrostomy $(\mathrm{n}=12)$ and cystoduodenostomy $(\mathrm{n}=2)$, with clinical follow-up using abdominal computed tomography for up to 51 months. Retrograde endoscopic cholangiopancreatography was attempted in all cases to study the pancreatic duct and classify the cysts. Results - There were 10 cases ( $71.5 \%)$ of chronic pancreatitis that had become acute through alcohol abuse and 4 (28.5\%) that had become acute through biliary disorders. Both types of endoscopic drainage (cystogastrostomy and cystoduodenostomy) were effective. There was no change in the therapeutic management proposed. Migration of the orthesis into the pseudocyst at the time of insertion (two cases) was the principal complication, and these could be removed during the same operation, by means of a Dormia basket, with the aid of fluoroscopy. There has so far not been any mortality or relapse. The mean hospital stay was 3 days. Conclusion - Transmural endoscopic drainage was an efficacious form of therapy, presenting a low complication rate and no mortality, and only requiring a short stay in hospital.

HEADINGS - Pancreatic pseudocyst. Drainage. Stents. Endoscopy, gastrointestinal.

\section{REFERÊNCIAS}

1. Adler DG, Pearson RK, Baron TH. Endoscopic drainage of a pancreatic pseudocys in a symptomatic patient with subtotal gastrectomy and Roux-en-Y anastomosis. Gastrointest Endosc. 2003;57:787-90.

2. Barthet M, Sahel J, Bodiou-Bertei C, Bernard JP. Endoscopic transpapillary drainage of pancreatic pseudocysts. Gastrointest Endosc. 1995;42:273-5

3. Beckingham IJ, Krige JE, Bornman PC, Terblanche J. Long term outcome of endoscopic drainage of pancreatic pseudocysts. Am J Gastroenterol. 1999;94:8-9.

4. Binmoeller KF, Seifert H, Walter A, Soehendra N. Transpapillary and transmural drainage of pancreatic pseudocysts. Gastrointest Endosc. 1995;42:219-24.

5. Cahen D, Rauws E, Fockens P, Weverling G, Huibregtse K, Bruno M. Endoscopic drainage of pancreatic pseudocysts: log-term outcome and procedural factors associated with safe and successful treatment. Endoscopy. 2005;37:977-83.

6. Catalano MF, Geenen JE, Schmaiz MJ, Johnson GK, Dean RS, Hogan WJ. Treatment of pancreatic pseudocysts with ductal communication by transpapillary pancreatic duct endoprothesis. Gastrointest Endosc. 1995;42:214-8.

7. Conwell DL. Motion-pancreatic endoscopy is useful for pain of chronic pancreatitis arguments against the motion. Can J Gastroenterol. 2003;17:61-3.

8. Cremer M, Devière J, Engelholm L. Endoscopic management of cysts and pseudocysts in chronic pancreatitis: long-term follow-up after 7 years of experience. Gastrointes Endosc. 1989;35:1-9.

9. Dani R, Martins GM. Pancreatite crônica - tratamento endoscópico. Rev Bras Pâncreas. 2004;16:32-9.

10. Dani R. Pseudocistos da pancreatite aguda (cistos pós-necróticos). Rev Bras Pâncreas. 2004;15:62-7.

11. Fainstein C. Drenagem externa percutânea de pseudocisto de pâncreas [dissertação] Niterói: Universidade Federal Fluminense; 1997.
12. Fockens P, Johnson TG, van Dullemen HM, Huibregtse K, Tytgat GN. Endosonographic imaging of pancreatic pseudocysts before endoscopic transmural drainage. Gastrointest Endosc. 1997;46:412-6.

13. Gitanjali V, Dani R. Pseudocistos da pancreatite aguda (cistos pós-necróticos). Rev Bras Pâncreas 2004; 15(4):62-7.

14. Grimm H, Binoeller KF, Soehendra N. Endosonography-guided drainage of a pancreatic pseudocysts. Gastrointest Endosc. 1992;38:170-1.

15. Kozarek RA. Endoscopic treatment of pancreatic pseudocysts. Gastrointest Endosc Clin N Am. 1997;7:271-83.

16. Lawson JM, Baillie J. Endoscopic therapy for pancreatic pseudocysts. Gastrointest Endosc Clin N Am. 1995;5:181-93.

17. Della Libera E, Siqueira ES, Morais M, Rohr MR, Brant CQ, Ardengh JC, Ferrari AP. Pancreatic pseudocysts transpapillary and transmural drainage. HPB Surg. 2000;11:333-8.

18. O'Malley VP, Cannon JP, Postier RG. Pancreatic pseudocysts: cause, therapy, and results. Am J Surg. 1985;150:680-2.

19. Schutz SM, Leung JW. Pancreatic endotherapy for pseudocysts and fluid collections. Gastrointes Endosc. 2002;56:150-2.

20. Sharma SS, Bhargawa N, Govil A. Endoscopic management of pancreatic pseudocyst: a long-term follow-up. Endoscopy. 2002;34:203-7.

21. Smits ME, Rauws EAJ, Tytgat GNJ, Huibretse K. The efficacy of endoscopic treatment of pancreatic pseudocysts. Gastrointest Endosc. 1995;442:202-7.

22. Stephen ES. Endoscopic management of pancreatic pseudocysts. Surg Clin N Am. 2001;81:405-10. 